\title{
Plasmodium phylogenetic profile: an assessment of a predictive tool for protein-protein interactions
}

\section{G. Sferra ${ }^{\bowtie}$, D. Santoni ${ }^{2}$, E. Pizzi ${ }^{1}$}

Istituto Superiore di Sanità, Roma, Italy

${ }^{2}$ Istituto di Analisi dei Sistemi ed Informatica, CNR, Italy

\section{Motivations}

Prediction of protein-protein interactions (PPIs) is a crucial goal for bioinformatics and the increasing availability of sequenced genomes support this challenge. One of the computational tools mostly used for this aim is the phylogenetic profiling, based on the co-conserved proteins identification by local alignments. The master idea is that co-evolving proteins share the same phylogenetic profile and can be grouped functionally. The field of parasite biology received a powerful improvement from post-genomics data. Several approaches have been utilized to predict PPIs for Plasmodium falciparum, including phylogenetic profiling. In contrast, no information about this is available in the case of Plasmodium berghei, even though for this parasite a huge amount of data is now available especially for mutant phenotypes.

\section{Methods}

A new strategy has been developed to derive phylogenetic profiling. The critical steps of this strategy are: 1) genomes selection; 2) global vs local alignments comparison; 3) mutual information vs correlation coefficients calculation. Agreeing with specific criteria, 774 reference organisms, on 1133 available on Eggnog database (January 2012), were included in the study, a global alignment algorithm, over the mostly used local one, was used to perform proteins identification across the genomes, the Mutual Information and the Correlation Coefficient were calculated and the results were compared. Escherichia coli K12 before, suitable for the assessment of the method, and P. berghei later, were used as target organisms.

\section{Results}

This analysis offers a new bioinformatical strategy to derive phylogenetic profile of an organism, highlighting on guidelines for the genomes selection, on the performance of different alignment algorithms and mathematical procedures. Moreover, strongly improve the knowledge about P. berghei and offers a new tool for evolution understanding and functional grouping of the proteins of this important biological model. 